\title{
Editorial
}

\section{Resource Management in Virtualized Clouds}

\author{
Ligang He, ${ }^{1}$ Laurence T. Yang, ${ }^{2}$ Zhihui $\mathrm{Du}^{3}$ and Florin Pop ${ }^{4}$ \\ ${ }^{1}$ University of Warwick, Coventry, UK \\ ${ }^{2}$ St. Francis Xavier University, Antigonish, NS, Canada \\ ${ }^{3}$ Tsinghua University, Beijing, China \\ ${ }^{4}$ University Politehnica of Bucharest, Bucharest, Romania \\ Correspondence should be addressed to Ligang He; liganghe@dcs.warwick.ac.uk
}

Received 10 October 2016; Accepted 11 October 2016

Copyright (C) 2016 Ligang He et al. This is an open access article distributed under the Creative Commons Attribution License, which permits unrestricted use, distribution, and reproduction in any medium, provided the original work is properly cited.

Resource management is of paramount importance in achieving high performance in Cloud environments. Different from traditional parallel and distributed systems, resource virtualization is a key feature in Cloud systems. Although researchers have been developing various strategies and techniques to address the resource management issues in virtualized Clouds, they, as this special issue shows, still face many new research challenges.

This special issue aims to report the latest scientific advances in resource management techniques for virtualized Clouds, especially on the topics including VM consolidation strategies, scheduling techniques, techniques for managing various types of virtualized resource, and VM management for various types of application. This issue received 47 high quality submissions. After the rigorous review process, 23 papers were accepted in this issue, documenting the relevant research from China, Spain, USA, Australia, Korea, Mexico, Luxembourg, Russia, France, and so forth.

The research presented in these 23 papers broadly covers the interesting scope of this special issue. Among these papers, scheduling strategies are proposed for various types of application and platform. W. Zheng et al. from Xiamen University in China proposed a randomization approach for scheduling stochastic workflows. J. M. Cortés-Mendoza et al. from CICESE Research Center in Mexico developed a new method for scheduling VoIP (Voice over Internet Protocol) tasks in Clouds. W. Lin et al. from South China University of Technology in China designed a task scheduling algorithm for heterogeneous virtual clusters. J. P. Orellana et al. from University of Castilla-La Mancha in Spain investigated scheduling strategies for FPGA devices equipped in Clouds.
Different resource management techniques are also investigated for virtualized Clouds. For example, a novel taskclassification-based VM consolidation method was proposed by $\mathrm{H}$. Choi et al. from Korea University in Korea. T. Chen et al. from Shanghai Jiao Tong University in China developed a new VM placement method by taking traffic balancing into account.

In addition to managing computation resources, new techniques are investigated for managing other types of resource. As an example, the dynamic allocation of network resources was studied by C.-H. Hong et al. from Korea University in Korea. J. No et al. at Sejong University in Korea implemented the cache management scheme for virtualized I/O resources.

Finally, although virtual machine is still a popular medium for providing the running environments for tasks and services, other types of virtualization technologies are emerging. B. Memishi et al. from Universidad Politécnica de Madrid in Spain investigated the resource allocation based on containers, a fine-grain alternative virtualization technology to virtual machines. W. Ai et al. from Hunan University in China proposed a novel metric to measure the elasticity of virtualized resources. For this special issue, we are able to select excellent papers providing a range of methods on the theme of the special issue.

\section{Acknowledgments}

We would like to thank the authors for contributing to our special issue. We also thank the reviewers for taking their 
valuable time to review and provide constructive comments to the authors.

Ligang $\mathrm{He}$

Laurence T. Yang

Zhihui Du

Florin Pop 

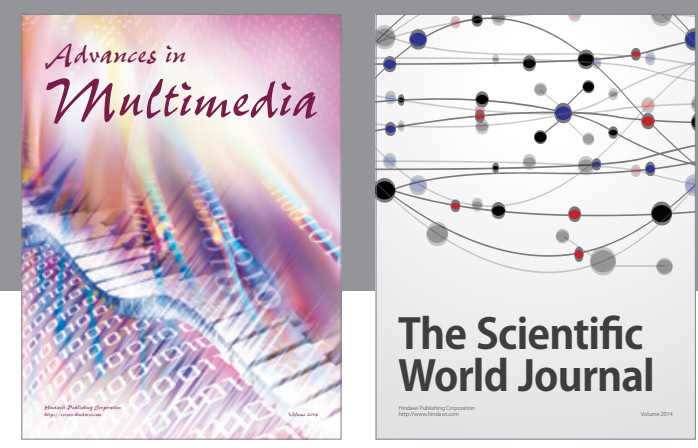

The Scientific World Journal
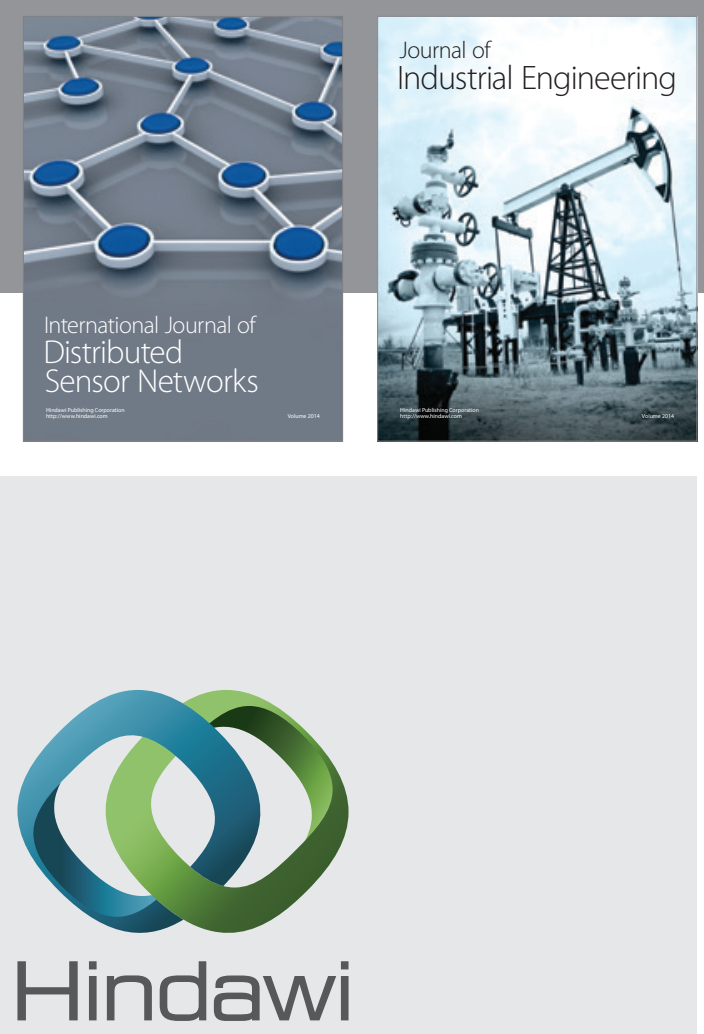

Submit your manuscripts at

http://www.hindawi.com

\section{Computer Networks} and Communications
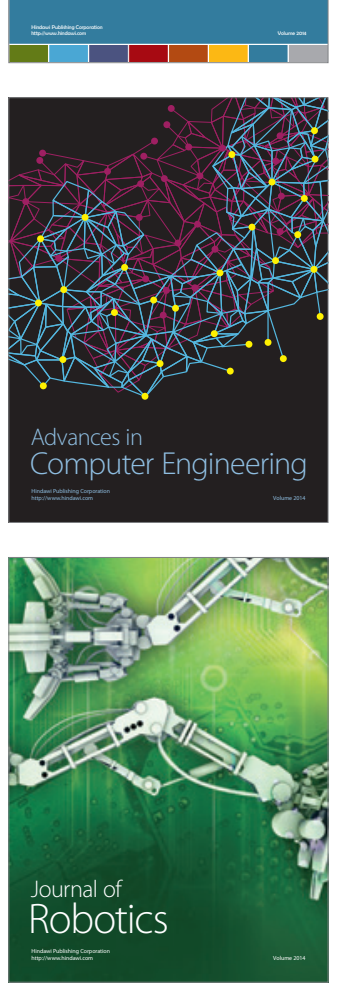
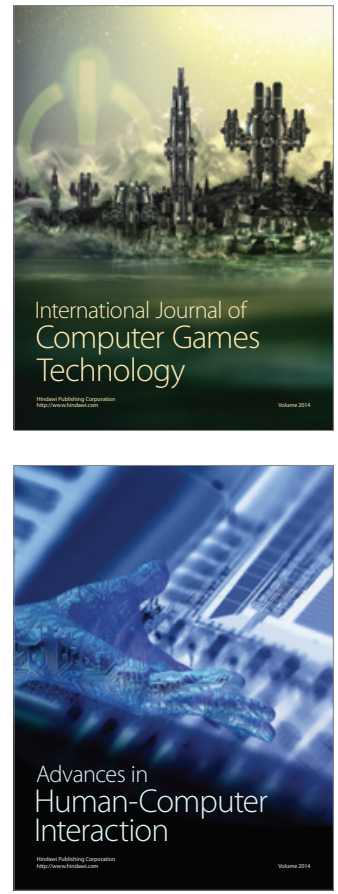
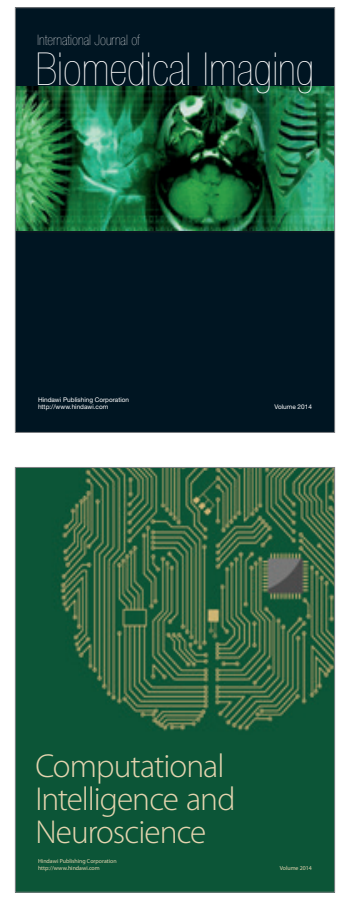
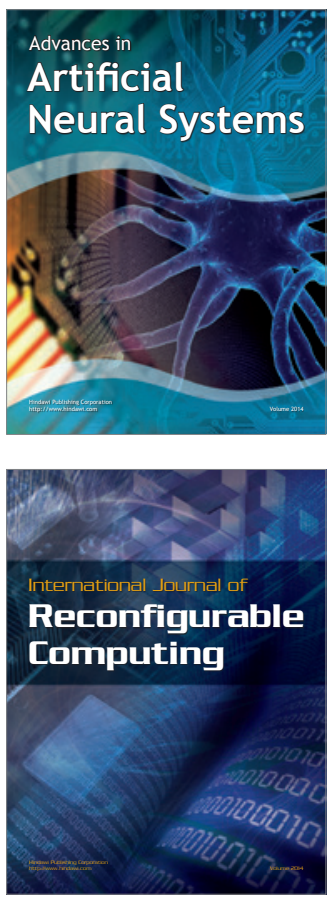
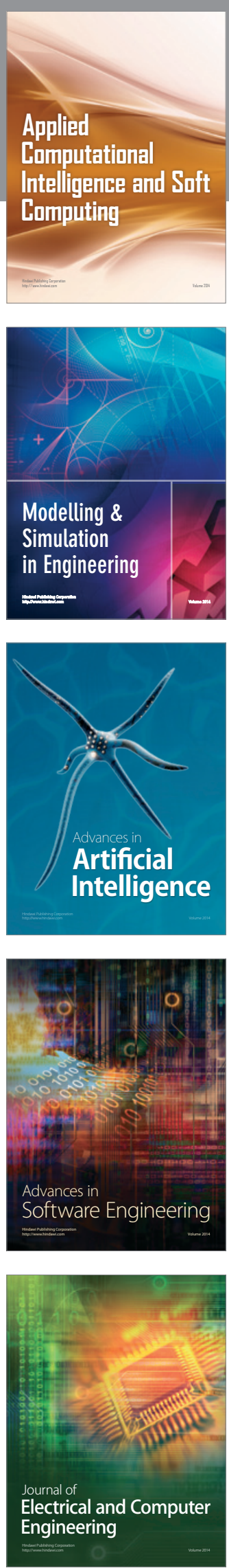